\title{
Evidências de Validação de uma Medida de Características Pessoais de Regulação das Emoções
}

\author{
Validation Evidences of a Measure of Personal Characteristics \\ of Emotional Regulation
}

\author{
Sonia Maria Guedes Gondim*, a , Cícero Roberto Pereira ${ }^{b}$, Ana Lúcia Teixeira Hirschle ${ }^{a}$, \\ Emanuel M. Silva Palma ${ }^{a}$, Gisele Debiasi Alberton ${ }^{a}$, Juliana Paranhos ${ }^{a}$, Vitor Santana ${ }^{a}$ \\ \& Wilma Raquel Barbosa Ribeiro ${ }^{a}$ \\ ${ }^{a}$ Universidade Federal da Bahia, Salvador, BA, Brasil \\ $\&{ }^{b}$ Universidade Federal da Paraíba, João Pessoa, PB, Brasil
}

\begin{abstract}
Resumo
Este artigo apresenta o Emotion Regulation Profile (ERP) como um instrumento para medir diferenças individuais na regulação de emoções. O ERP é constituído por 15 cenários que apresentam situações características de eventos da vida cotidiana desprazerosos (nove cenários) ou prazerosos (seis cenários). Cada cenário avalia a quantidade de respostas adaptativas/funcionais e desadaptativas/ disfuncionais dos participantes. $\mathrm{O}$ instrumento foi aplicado a uma amostra de 686 pessoas entre $18 \mathrm{e}$ 51 anos de idade. A amostra foi sudividida em dois grupos de 343 participantes para procedimentos de análises fatoriais exploratórias e confirmatórias. Os resultados da análise de componentes principais (primeira amostra) corroborou a estrutura bifatorial identificada no estudo original da medida, isto é, um fator de regulação das emoções negativas (down regulation) e um fator de regulação das emoções positivas (up regulation). Além disso, um conjunto de análises fatoriais confirmatórias (segunda amostra) mostrou que a estrutura bifatorial proposta teve um bom ajustamento aos dados. Por fim, foi possível propor uma versão reduzida do ERP com seis cenários (três que descrevem situações desprazerosas e três prazerosas) para avaliar adequadamente as diferentes estratégias usadas pelas pessoas na regulação de suas emoções.

Palavras-chave: Emoções, regulação das emoções, medida de gerenciamento afetivo.
\end{abstract}

\begin{abstract}
This article presents the Emotion Regulation Profile (ERP) as an instrument for measuring individual differences in the regulation of emotions. The ERP consists of 15 vignettes that portray everyday life situations describing pleasant (nine vignettes) and unpleasant (six vignettes) events. Each vignette assesses the number of adaptive/functional and maladaptive/dysfunctional responses chosen by the participants. The instrument was administered to a sample of 686 people between the ages of 18 and 51. The sample was divided into two groups of 343 participants each so that exploratory and confirmatory factor analysis could be conducted. Results from the principal component analysis (first sample) corroborated the bifactorial structure identified in the original study of the measure, that is, a regulation of negative emotion factor (down regulation) and a regulation of positive emotion factor (up regulation). Furthermore, a set of confirmatory factor analysis (second sample) revealed that the proposed bifactorial structure showed good fit indices. Finally, it was possible to propose a reduced version of the ERP with six vignettes (three describing pleasant events and three describing unpleasant events) to adequately assess the different strategies people use when regulating their emotions. Keywords: Emotions, emotion regulation, affective management measure.
\end{abstract}

A regulação emocional envolve um conjunto de processos neurofisiológicos, cognitivos e comportamentais, conscientes (emoções sociais e secundárias) e automáticos

\footnotetext{
"Endereço para correspondência: Universidade Federal da Bahia, Rua Aristides Novis, 197, Federação, Salvador, BA, Brasil 40210-630. E-mail: sggondim@gmail. com, cicero.pereira@ics.ul.pt, analuciate@gmail.com, emanuelmssilva@gmail.com, agdebiasi@yahoo.com. br,julipmb@gmail.com, vitor_santana23@hotmail.com ewrmima@hotmail.com
}

(emoções básicas). O constructo tem sido objeto de análise em diversos domínios, dentre os quais se destacam o educacional (Zhao, 2011), o do trabalho (Diefendorff, Croyle, \& Gosserand, 2005; Grandey, 2000; Hochschild, 1983), o do desenvolvimento infantil (Fabes et al., 1999) e o da atividade clínica (Mennin, 2006). A psicologia do desenvolvimento foi uma das principais subáreas da psicologia a dedicar-se à compreensão da dinâmica de cada emoção e à identificação das respostas emocionais mais adaptativas às diversas situações agradáveis e desagradá- 
veis da vida cotidiana (Aldao, 2013). A constatação foi que a expressão de uma emoção positiva (e.g., alegria) ou negativa (e.g., tristeza) não é suficiente para que se defina a qualidade da resposta emocional em termos de seus efeitos adaptativos ou desadaptativos (Izard, 2010; Izard et al., 2011). Em outras palavras, dependendo da situação e do tipo de resposta emocional (positiva ou negativa) exigida em cada contexto, torna-se necessário mobilizar estratégias diferenciadas para assegurar melhor adaptação da resposta emocional à situação social.

Neste sentido, uma resposta emocional não seria em si positiva ou negativa, mas dependeria do contexto de manifestação, tornando importantes os processos regulatórios e o uso de estratégias de gerenciamento de emoções (e.g. Gross, 1998; Gross \& Thompson, 2007; Níven, Totterdell, Stride, \& Holman, 2011). Nelis, Quoidbach, Hansenne e Mikolajczak (2011) propuseram o Emotion Regulation Profile (ERP), um instrumento de medida da regulação emocional que destaca o papel central que as características do contexto social desempenham nas estratégias usadas pelas pessoas para lidar com emoções positivas e negativas. Nelis et al. (2011) construíram a medida supondo haver dois modos básicos de regulação das emoções, um que responde pela diminuição dos efeitos indesejáveis das emoções negativas e outro que responde por potencializar os efeitos desejáveis das emoções positivas. O ERP tem em consideração esses dois modos e avalia em que medida as pessoas são capazes de aumentar os efeitos de suas emoções positivas (up regulation) ou reduzir os efeitos indesejáveis da expressão de suas emoções negativas (down regulation) em situações em que se espera que essas emoções sejam reguladas.

No presente artigo apresentam-se as evidências de validação do ERP em uma amostra brasileira. Além de apresentar as propriedades psicométricas desse instrumento, propõe-se uma versão-reduzida do ERP em que foram avaliadas as dimensões do conceito e submetidas a teste por meio de um conjunto de análise fatoriais confirmatórias. A necessidade de uma versão reduzida da medida deve-se ao fato de a versão completa ser demasiado extensa (15 cenários com oito estratégias de regulação em cada um deles), dificultando o uso de instrumentos adicionais em estudos que visem correlacionar a regulação emocional com outras variáveis.

\section{Analisando o Processo de Regulação Emocional: O que Tenta Medir o Emotion Regulation Profile-Revised (ERP)}

O Emotion Regulation Profile (ERP) é uma medida de diferenças individuais na regulação de emoções (Nelis et al., 2011). Trata-se de uma medida sendo composta por cenários selecionados pelos autores a partir de uma ampla revisão da literatura sobre regulação emocional de 1995 a 2008. Cada um deles descreve diferentes tipos de situações que envolvem a ativação de emoções positivas e negativas (ver exemplos dos cenários nas Figuras 1 e 2) sendo seguido de oito possíveis reações ao evento, sendo elas, quatro consideradas adaptativas e funcionais e, ao contrário, quatro consideradas desadapativas e disfuncionais. Em cada cenário, o respondente pode eleger quantas estratégias julgar serem necessárias para melhor representar seu modo de agir na situação apresentada.

As estratégias de regulação das emoções positivas e negativas identificadas pelos autores são apresentadas a seguir (Nelis et al., 2011). Nos cenários que eliciam emoções negativas, as quatro estratégias funcionais de regulação destas emoções (diminuem os efeitos das emoções negativas) são:

1. Modificação da Situação, que envolve ação direta para transformar a situação e modificar seu impacto emocional;

2. Reorientação da Atenção, que implica desvio do foco da atenção para modificar o sentimento;

3. Reavaliação Positiva da Situação, que se refere à mudança do pensamento sobre a situação inicialmente negativa; e

4. Expressão das Emoções, que envolve o compartilhamento das emoções negativas com os outros.

Já as quatro estratégias disfuncionais para lidar com as emoções negativas (aumentam os efeitos danosos das emoções negativas) são:

5. Desamparo Aprendido, que se refere ao comportamento passivo acompanhado de um sentimento de impotência;

6. Ruminação, que envolve excesso de atenção a sentimentos e pensamentos associados a eventos negativos, aumentando a duração e intensidade das emoções negativas;

7. Abuso de Substâncias, que envolve uso de bebidas alcoólicas, ansiolíticos ou drogas para se esquivar de eventos adversos na tentativa de evitar mais consequências emocionais negativas; $\mathrm{e}$

8. Reação Impulsiva (Acting out), que se refere à ação irrefletida desencadeada por forte intensidade emocional.

Em relação aos cenários em que estão envolvidas emoções positivas, pode-se optar entre oito diferentes estratégias de regulação. Quatro são consideradas adaptativas (aumentam os efeitos das emoções positivas):

1. Manifestação do Comportamento, que envolve a expressão de emoções positivas acompanhada de comportamentos não verbais sintonizados;

2. Saborear o Momento Presente, que se refere ao foco da atenção nas experiências momentâneas agradáveis;

3. Capitalização, que inclui comportamentos como a comunicação e a celebração de eventos positivos com outros; e

4. Viagem mental positiva, que se refere ao resgate de lembranças positivas ou antecipação de eventos positivos.

Finalmente, as outras quatro estratégias são consideradas desadaptativas (diminuem os efeitos das emoções positivas): 
Gondim, S. M. G., Pereira, C. R., Hirschle, A. L. T., Palma, E. M. S., Alberton, G. D., Paranhos, J., Santana, V. \& Ribeiro, W. R. B. (2015). Evidências de Validação de uma Medida de Características Pessoais de Regulação das Emoções.

5. Inibição da Expressão Emocional, que envolve a supressão de emoções positivas;

6. Preocupação Excessiva, que se refere à tendência a desviar o foco do evento positivo e a se preocupar constantemente com outras coisas;

7. Identificação de Falhas, que envolve o foco em potenciais aspectos negativos da situação positiva ou no que poderia ser melhor; $\mathrm{e}$

8. Viagem Mental Negativa, que se refere à ênfase na atribuição externa de eventos positivos e na antecipação negativa de consequências futuras.

Os autores da medida original realizaram análises exploratórias recorrendo a um conjunto de análise de componentes principais que mostraram a organização dos cenários em dois fatores: redução dos efeitos indesejáveis das emoções negativas quando ativadas (down regulation) e aumento dos benefícios das emoções positivas quando ativadas (up regulation). Essa solução explicava $42 \%$ da variância total das respostas dos participantes ao conjunto dos 15 cenários. A força da correlação entre os dois fatores apresentou magnitude moderada $(r=0,49$, $p<0,001)$ e mostrou-se coerente com os pressupostos teóricos da medida. Assim, a ERP avalia dois fatores: um que identifica a tendência dos indivíduos para agirem de modo adaptativo em termos de regulação emocional quando potencializam os benefícios das emoções positivas (uso de estratégias adaptativas - up regulation); e um que avalia a habilidade das pessoas para minimizarem o impacto indesejado das emoções negativas (uso de estratégias funcionais - down regulation).

\section{Método}

\section{Participantes}

A amostra deste estudo foi não aleatória, tendo sido usada a estratégia do snowball. Participaram do estudo 686 pessoas, sendo a maioria $(72,6 \%)$ do sexo feminino. As idades variaram de 18 a 51 anos, com média de $26,64(D P=8,83)$. Quanto ao nível educacional, a maioria $(55,3 \%)$ ainda estava cursando o ensino superior, enquanto $40 \%$ já o haviam concluído. Somente $0,3 \%$ dos participantes possuía apenas o ensino fundamental completo. Em termos de atuação profissional, 53,6\% da amostra foi composta por estudantes e $39,8 \%$ de pessoas de nível superior com inserção profissional. Para proceder às análises, a amostra foi aleatoriamente dividida em duas partes, cada uma composta por 343 participantes. A primeira amostra foi usada para análise dos componentes principais e a segunda amostra para a análise fatorial confirmatória e a proposição do modelo reduzido. As duas amostras não apresentaram diferenças em relação à idade média, sexo e nível educacional.

\section{Instrumento}

O ERP é composto por 15 cenários, dos quais nove descrevem situações eliciadoras de respostas emocionais negativas (Figura 1) e seis descrevem situações eliciadoras de emoções positivas (Figura 2). As respostas emocionais negativas ativadas nos nove cenários com situações desagradáveis são: raiva (cenários 1 e 10), tristeza (cenários 4 e 11), medo (cenários 8 e 15), ciúme (cenário 6), vergonha (cenário 13) e culpa (cenário 3). As respostas emocionais positivas ativadas nos seis cenários prazerosos são: alegria (cenário 7), contentamento (cenário 2), admiração (cenário 9), excitação (cenário 5), gratidão (cenário 14) e orgulho (cenário 12). Para cada cenário, são oferecidas oito alternativas de respostas.

Nos cenários que descrevem situações prazerosas, são apresentadas quatro opções de respostas envolvendo estratégias adaptativas (manifestação do comportamento, saborear o momento presente, capitalização, viagem mental positiva) e quatro opções de resposta que se referem ao uso de estratégias consideradas desadaptativas (inibição da expressão emocional, preocupação excessiva, identificação de falhas e viagem mental negativa). No caso dos cenários que descrevem situações desagradáveis, são também apresentadas quatro opções de resposta que caracterizam estratégias funcionais (modificação da situação, reorientação da atenção, reavaliação positiva da situação, expressão das emoções) e quatro opções de respostas que descrevem estratégias disfuncionais (desamparo aprendido, ruminação, abuso de substâncias e reação impulsiva). Em cada uma das situações os participantes podem indicar quantos comportamentos julgam necessários para representarem a diversidade de suas reações. A diferença entre a quantidade de estratégias adaptativas e desadaptativas indica, em cada cenário, a capacidade do respondente em gerenciar adequadamente a expressão de suas emoções.

Em relação à precisão e às evidências de validade dos fatores que reúnem os itens da escala, o índice de confiabilidade global do instrumento foi satisfatório $(\alpha=0,84)$. Os dois fatores, redução dos efeitos indesejáveis das emoções negativas (down regulation) e potencialização dos benefícios das emoções positivas (up regulation) também mostraram consistência interna satisfatória $(\alpha=0,83$ e $\alpha=0,79$, respectivamente). Os autores também mostraram evidência de validade convergente e discriminante, revelando que os escores do ERP se diferenciavam do raciocínio não verbal e habilidades verbais e mostravam convergência com uma medida de inteligência emocional e várias dimensões de personalidade medidas com big-five. Foram encontradas também evidências preliminares de validade de critério e incremental, uma vez que o ERP mostrou-se ser um bom preditor de vários indicadores de bem-estar e saúde mental (Nelis et al., 2011).

\section{Procedimentos de Tradução do ERP}

Inicialmente, os itens do ERP foram traduzidos do francês (idioma oficial dos autores da escala original) para o português, por uma tradutora proficiente em ambos os idiomas. Foi realizada uma comparação dos itens entre a versão original e a versão traduzida para o português brasileiro, tendo sido levados em consideração os seguintes parâmetros: equivalência semântica (significados), 


\section{Cenário 7: Você passa um fim de semana romântico. O cenário é perfeito. Seu(sua) parceiro(a) é demais e você está se sentindo particularmente feliz.}

( ) Apesar de um fim de semana muito agradável, você não pode deixar de perceber alguns pontos negativos que impedem que a estadia seja perfeita. (Identificação de falhas)

( ) Você tenta desfrutar o momento, colocando todo o resto de lado. (Saborear o momento presente)

( )O fim de semana é perfeito. Isso é bom demais para ser verdade. Você teme que algum pensamento estrague esse momento. (Viagem mental negativa)

( )Você está rindo, brincando, abraçando seu/sua parceiro(a) ... Enfim, você se permite extravasar toda a sua alegria. (Manifestação do comportamento)

( )Quando está sozinho (a), você relembra os bons momentos juntos e / ou as razões pelas quais seu relacionamento é tão valioso. (Viagem mental positiva)

( )Você curte o momento, mas por razões diferentes (por exemplo, medo de ridículo, não é o seu estilo, culpa, ...) você tenta não se "deixar levar" e contém a sua alegria.

(Inibição da expresisão emocional)

( )Nos dias seguintes, você compartilha esse bom momento com pessoas próximas (ou escreve em seu diário). (Capitalização)

( )O fim de semana é perfeito. No entanto, você não pode deixar totalmente de lado as suas preocupações atuais (por exemplo, trabalho, família, ...). (Preocupação excessiva)

Figura 1. Exemplo de cenário de alegria com as opções de estratégias.

Cenário 8: Você tem que fazer uma apresentação oral para várias pessoas. Já fez isso antes e não se saiu muito bem. Você recebeu muitas críticas de sua apresentação. A ideia de reviver uma situação de exposição pública, dentro de poucos dias, $o$ aterroriza.

( ) Você tenta se distrair fazendo uma atividade que lhe é agradável. Você preparou a sua apresentação e espera o dia chegar como outro qualquer. (Reorientação da atenção)

( ) Você não para de pensar nisto, se concentra sobre o que poderia dar errado e se estressa até o dia da apresentação. (Ruminação)

( )Você compartilha seus medos e busca ao seu redor apoio e aconselhamento. (Expressão das emoções)

( )Você estabelece um plano de ação para ter mais chances de sucesso. Você define o problema e planeja diferentes soluções para se sentir mais seguro(a) (ensaio, relaxamento, informações sobre como melhorar a sua apresentação). (Modificação da situação)

( )Você diz que nunca vai chegar lá e se sente "um zero à esquerda". (Desamparo aprendido)

( Dias antes da apresentação, você usa substâncias relaxantes (por exemplo, álcool, maconha, medicamentos,...) para diminuir sua ansiedade. (Abuso de substâncias) ( )Você tenta ver o lado positivo da situação: será um bom exercício para você e, se as coisas derem errado, não será o fim do mundo! (Reavaliação positiva da situação) ( )Desde o anúncio da data de apresentação o estresse toma conta de você. Ele o paralisa e lhe impede de trabalhar na sua apresentação. Sendo possível, você encontra uma "excelente razão" que lhe impede de fazer a sua apresentação. (Reação impulsiva/Acting out)

Figura 2. Exemplo de cenário de medo com as opções de estratégias. 
Gondim, S. M. G., Pereira, C. R., Hirschle, A. L. T., Palma, E. M. S., Alberton, G. D., Paranhos, J., Santana, V. \& Ribeiro, W. R. B. (2015). Evidências de Validação de uma Medida de Características Pessoais de Regulação das Emoções.

equivalência idiomática (expressões compatíveis com o idioma a ser traduzido), equivalência cultural (situações pertinentes) e equivalência conceitual (Borsa, Damásio, \& Bandeira, 2012; Cassepp-Borges, Balbinotti, \& Teodoro, 2010; Pasquali, 2010). Posteriormente foi feita uma back translation para ser comparada com a versão original e avaliada a sua equivalência por outro tradutor francês. Para finalizar, solicitou-se a voluntários e estudantes universitários e profissionais de nível superior, que respondessem ao instrumento antes de dar início efetivo à coleta de dados. Não foi necessário nenhum ajuste adicional, o que permite assegurar a adequação semântica do ERP ao português falado no Brasil.

\section{Procedimento de Coleta de Dados}

O ERP foi aplicado de forma online por meio da plataforma EFS Survey. O estudo foi divulgado em salas de aulas, palestras dadas por membros do grupo de pesquisa, listas de $e$-mail de estudantes e profissionais, além de redes sociais disponíveis na internet. A concordância com o termo de consentimento livre e esclarecido foi uma exigência antes do início do preenchimento do instrumento.

\section{Procedimento de Tratamento e Análise dos Dados}

Conforme orientação dos autores proponentes do ERP, em cada cenário foram computados os comportamentos considerados adaptativos selecionados pelos participantes cuja soma poderia variar de 0 (nenhum comportamento adaptativo escolhido) a 4 (número máximo de comportamentos adaptativos escolhidos). Em seguida, contou-se a quantidade de comportamentos desadaptativos selecionados pelos participantes, cuja soma também poderia variar entre 0 (nenhum comportamento desadaptativo) e 4 (quantidade máxima de comportamentos desadaptativos escolhidos). Finalmente, os comportamentos desadaptativos foram subtraídos dos comportamentos adaptativos, de modo que cada cenário obteve um escore que variava de -4 (quantidade máxima de comportamentos disfuncionais) a +4 (quantidade máxima de comportamento adaptativos).

$\mathrm{Na}$ amostra 1 seguiu-se os mesmos procedimentos empregados pelos autores da ERP (Nelis et al., 2011) em que se procedeu a uma análise exploratória com o objetivo de identificar a estrutura fatorial da medida. A estrutura fatorial obtida na amostra 1 foi submetida a um conjunto de análises fatoriais confirmatórias na amosra 2, com o objetivo de testar a estrutura fatorial do ERP e também propor uma versão reduzida do instrumento. Os parâmetros foram estimados por meio do método da máxima verossimilhança aplicado à matriz de variância-covariância dos cenários. A adequação dos modelos fatoriais foi avaliada de acordo com os seguintes critérios (e.g., Byrne, 2010): razão entre o qui-quadrado e os graus de liberdade do modelo $\left(\chi^{2} / g l\right)$ menor do que 5; o CFI (Comparative Fit Index) e o AGFI (Adjusted Goodness-of-Fit Index) maiores do que 0,90; o RMSEA (Root Mean Square Error of Approximation) menor do que 0,05; e o AIC (Akaike Information Criterion) para fins de comparação de modelos fatoriais em que o modelo que apresenta menor valor de AIC tem um melhor ajustamento aos dados, por ser mais parcimonioso.

Foram testados dois modelos. O modelo base previa um fator geral latente diretamente relacionado a todos os 15 cenários. $O$ modelo alternativo previa dois fatores latentes de regulação emocional: um para emoção positiva (up regulation) e outro para emoção negativa (down regulation). Por fim, a versão reduzida foi proposta com base nos resultados do teste comparativo dos dois modelos anteriores. O objetivo foi propor uma versão reduzida do ERP com apenas 6 cenários ( 3 cenários positivos e 3 negativos), preservando as boas propriedades psicométricas obtidas na versão completa do instrumento. Os cenários da versão reduzida foram selecionados com base nos maiores pesos fatoriais, assegurando a inclusão de situações que eliciavam tanto emoções primárias quanto secundárias, positivas e negativas. Assim, para o fator de emoções negativas (down regulation) foram escolhidos os cenários de ciúme (cenário 6), tristeza (cenário 4) e medo (cenário 8), enquanto que para o fator de emoções positivas (up regulation) foram incluídos os cenários alegria (cenário 7), orgulho (cenário 12) e admiração (cenário 9; ver Tabela 1).

\section{Resultados}

A distribuição dos cenários não difere da distribuição normal em ambas as amostras. De maior importância, a análise dos resíduos associados a cada cenário indicou estarem normalmente distribuídos e não foram encontrados outliers na base de dados.

\section{Resultados da Amostra 1: Análise dos Componentes Principais}

Em acordo com a orientação do estudo original do instrumento, procedeu-se a uma análise dos componentes principais com rotação promax com o objetivo de identificar componentes psicológicos básicos responsáveis pela organização dos comportamentos nos 15 cenários. Os resultados permitiram extrair dois fatores, sendo um referente aos cenários que ativam emoções positivas (up regulation) e outro aos cenários que ativam emoções negativas (down regulation), tal como previsto no estudo original. No entanto, dos 15 cenários da medida original, três apresentaram carga fatorial abaixo de 0,40 ou saturaram no fator oposto aos previsto por Nelis et al. (2011): o cenário 13 (vergonha, com carga -0,51 no fator 2 e -0,32 no fator 1 ), cenário 15 (medo, com carga 0,23 no fator $2 \mathrm{e}$ $-0,70$ no fator 1 ), e o cenário 14 (gratidão, com carga 0,13 no fator 1 e 0,01 no fator 2). Como havia outras situações que mediam situações eliciadoras de emoções primárias e secundárias positivas e negativas, e a exclusão dos três cenários revelou melhoria nos indicadores de confiabilidade (Alfa de Cronbach) dos dois fatores, optou-se pela exclusão dos três cenários. Após a retirada, procedeu-se uma vez mais à análise dos componentes principais apenas com os 12 cenários e os resultados encontram-se apresentados na Tabela 1 . 
Psychology/Psicologia: Reflexão e Crítica, 28(4), 659-667.

Tabela 1

Cargas Fatoriais, Fiabilidade e Estatística Descritiva dos Cenários em Cada Fator Medido pelo ERP

\begin{tabular}{lcccc}
\hline \multicolumn{1}{c}{ Cenários } & $\begin{array}{c}\text { Regulação ascendente para } \\
\text { emoções positivas ativadas } \\
(\text { Up regulation) }\end{array}$ & $\begin{array}{c}\text { Regulação descendente para } \\
\text { emoçoses negativas ativadas } \\
\text { (Down regulation })\end{array}$ & Assimetria & Achatamento \\
\hline Cenário 7 (alegria) & $\mathbf{0 , 7 7}$ & $-0,09$ & $-0,22$ & $-0,75$ \\
Cenário 9 (admiração) & $\mathbf{0 , 7 3}$ & 0,00 & $-0,22$ & $-0,73$ \\
Cenário 5 (excitação) & $\mathbf{0 , 7 1}$ & $-0,17$ & $-0,35$ & $-0,12$ \\
Cenário 12 (orgulho) & $\mathbf{0 , 6 8}$ & 0,09 & $-0,22$ & $-0,26$ \\
Cenário 2 (contente) & $\mathbf{0 , 5 9}$ & 0,17 & $-0,23$ & $-0,14$ \\
Cenário 4 (tristeza) & $-0,06$ & $\mathbf{0 , 7 2}$ & $-0,26$ & $-0,40$ \\
Cenário 6 (ciume) & $-0,17$ & $\mathbf{0 , 7 0}$ & $-0,28$ & 0,09 \\
Cenário 8 (medo) & 0,17 & $\mathbf{0 , 6 1}$ & $-0,14$ & $-0,42$ \\
Cenário 3 (culpa) & $-0,31$ & $\mathbf{0 , 5 9}$ & $-0,39$ & 0,38 \\
Cenário 11 (tristeza) & 0,26 & $\mathbf{0 , 5 0}$ & $-0,35$ & $-0,40$ \\
Cenário 1 (raiva) & $-0,14$ & $\mathbf{0 , 4 1}$ & $-0,16$ & $-0,43$ \\
Cenário 10 (raiva) & 0,21 & $\mathbf{0 , 3 6}$ & 0,24 & $-0,44$ \\
Alfa de Cronbach & 0,73 & $\mathbf{0 , 6 7}$ & & \\
Número de itens & 5 & 7 & & \\
Média dos fatores & 0,69 & 0,55 & & \\
Eingenvalues & 3,34 & 1,53 & & \\
Variância Explicada & $27,8 \%$ & $12,7 \%$ & & \\
\hline
\end{tabular}

Nota. Os ajustes em negrito destacam as cargas fatoriais aceitáveis para saturação em cada fator.

Os resultados indicam que as médias das cargas fatoriais situam-se entre 0,55 (emoções negativas) e 0,69 (emoções positivas), explicando $40,5 \%$ da variância. Os indicadores são semelhantes aos encontrados no estudo original. A correlação entre os dois fatores encontradas pelos autores proponentes da medida foi de $(r=0,49$, $p<0,001)$ e na amostra brasileira obteve-se uma correlação de $(r=0,50 ; p<0,001)$. Portanto, os resultados desta análise exploratória permitem propor uma estrutura fatorial de dois tipos de regulação emocional, que organizam os comportamentos selecionados pelos participantes em 12 dos 15 cenários inicialmente apresentados.

\section{Resultados da Amostra 2: Análise Fatorial Confirmatória}

A Figura 3 apresenta os parâmetros estimados de acordo com o modelo fatorial proposto. Todos os pesos fatoriais são significativos e variam de 0,28 a 0,66 no fator que representa a regulação de emoções negativas (down regulation) e de 0,50 a 0,67 no fator da regulação das emoções positivas (up regulation). A variação observada no fator das emoções negativas indica que nem todos os comportamentos selecionados pelos participantes representam fidedignamente a regulação das emoções negativas, especialmente o cenário 1 .

A análise da adequação do modelo indica um ajustamento aos dados muito bom da estrutura bi-fatorial proposta. Além disso, comparou-se este modelo com um modelo alternativo em que todos os cenários saturam num único fator geral. A comparação entre os dois modelos indicou que o modelo bifatorial proposto ajusta-se significativamente melhor aos dados e, portanto, descreve melhor os comportamentos que representam a regulação emocional. A Tabela 2 apresenta os resultados da comparação dos dois modelos testados.

Uma vez definido que o modelo de dois fatores é o de melhor ajuste, procedeu-se a uma nova análise fatorial confirmatória com o modelo reduzido proposto. A Figura 4 apresenta os parâmetros estimados de acordo com o modelo fatorial proposto.

Nesta solução, todos os cenários foram significativos e saturaram fortemente no seu respectivo fator o que indica que esses cenários representavam de forma adequada 
Gondim, S. M. G., Pereira, C. R., Hirschle, A. L. T., Palma, E. M. S., Alberton, G. D., Paranhos, J., Santana, V. \& Ribeiro, W. R. B. (2015). Evidências de Validação de uma Medida de Características Pessoais de Regulação das Emoções.

a regulação de emoções negativas (down regulation) e a regulação das emoções positivas (up regulation). De igual importância foi o fato de o modelo proposto ter apresentado um excelente ajuste aos dados, conforme se observa na Tabela 3. A comparação entre o modelo bifatorial proposto com um modelo em que se prevê apenas um Fator Geral indicou que a melhor solução foi, de fato, o modelo com os dois fatores correlacionados. Cada fator apresentando consistência interna satisfatória: regulação de emoções positivas alfa $=0,60$; regulação de emoções negativas alfa $=0,60$.

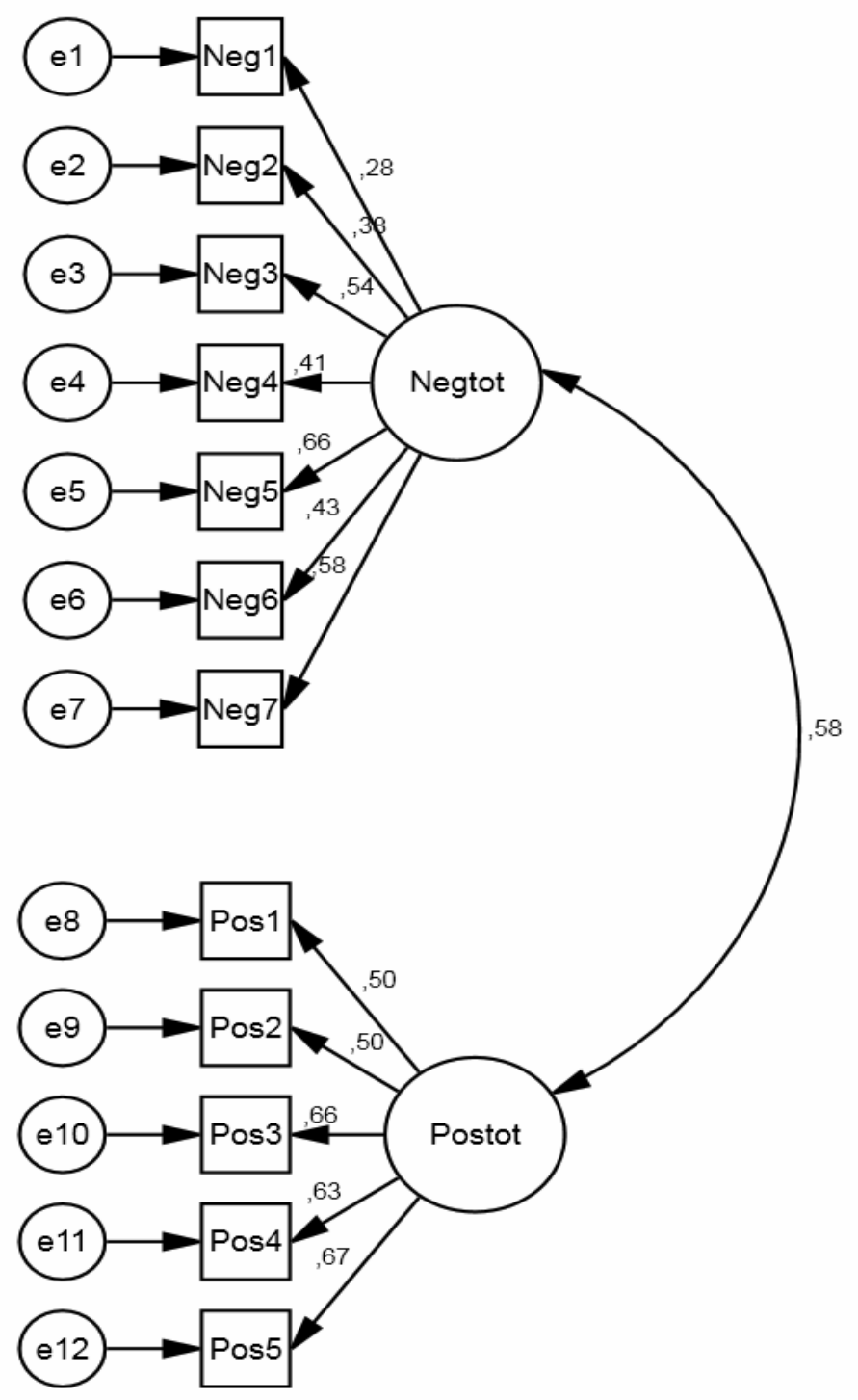

Figura 3. Modelo bifatorial de regulação: emoções positivas e emoções negativas.

Tabela 2

Indicadores de Ajustes dos Modelos Testados

\begin{tabular}{lcccccccc}
\hline Modelo & $\chi^{2}$ & $G L$ & $\chi^{2} / g l$ & RMSEA & IC95\% & GFI & CFI & BIC \\
\hline ERP-FG & 291,07 & 54 & 5,39 & 0,08 & $0,071 / 0,089$ & 0,91 & 0,81 & 447,71 \\
ERP_2F & 101,41 & 53 & 1,91 & 0,04 & $0,026 / 0,047$ & 0,97 & 0,96 & 264,58 \\
\hline
\end{tabular}

Nota . ERP-FG= Modelo fator geral, ERP_2F=Modelo com dois fatores: up regulation (5 itens), down regulation (7 itens). 


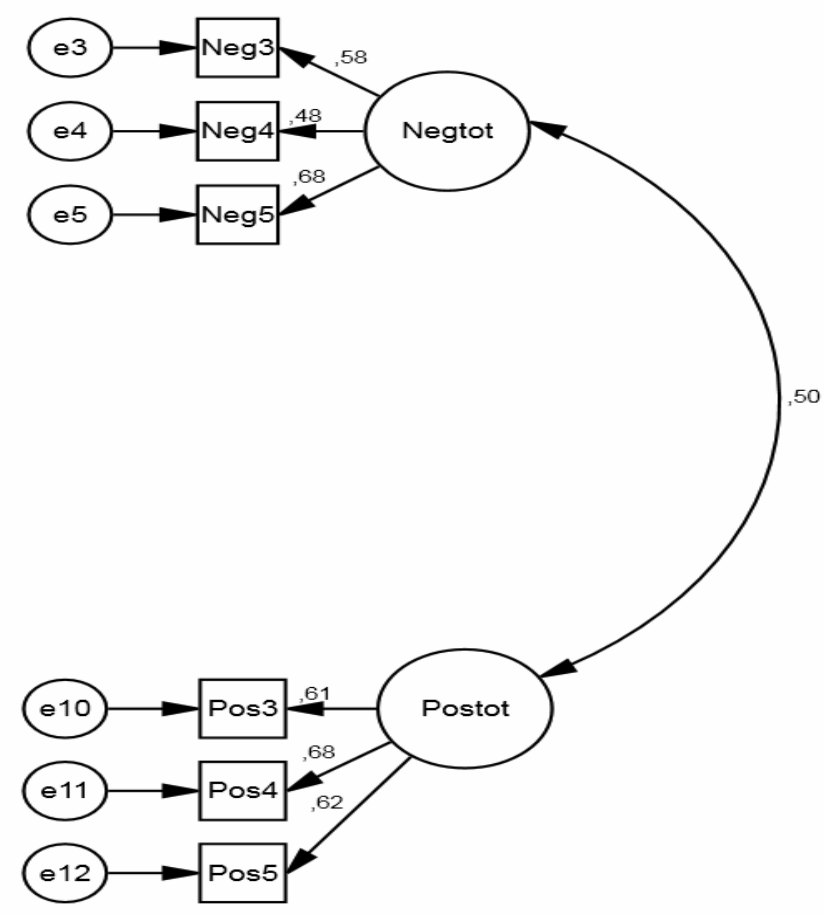

Figura 4. Versão reduzida da medida de ERP - perfil de regulação emocional.

Tabela 3

Indicadores de Ajuste da Versão Reduzida do ERP

\begin{tabular}{lcccccccc}
\hline Modelo & $\chi^{2}$ & $G L$ & $\chi^{2} / g l$ & RMSEA & IC95\% & GFI & CFI & BIC \\
\hline ERP_R & 11,29 & 8 & 1,4 & 0,03 & $0,00 / 0,074$ & 0,99 & 0,99 & 88,26 \\
ERP_FG_R & 76,62 & 9 & 8,51 & 0,14 & $0,11 / 0,17$ & 0,92 & 0,79 & 147,68 \\
\hline
\end{tabular}

Nota. ERP_FG_R: modelo comum com um fator geral e 3 itens de emoções negativas e 3 itens de emoções positivas; ERP_R versão reduzida, up regulation (3 itens) e down regulation (3 itens). A versão reduzida do ERP encontra-se disponível mediante solicitação aos autores.

\section{Discussão}

Este estudo apresentou evidências de validação do ERP em sua versão completa e reduzida em uma amostra de participantes brasileiros. Os resultados da análise dos componentes principais foram similares aos encontrados no estudo original de Nelis et al. (2011). Foram extraídos dois fatores, refletindo duas formas de regular as emoções, uma que visa aumentar os efeitos adaptativos das emoções positivas ativadas em situações agradáveis (up regulation) e outra que objetiva diminuir as consequências nocivas de emoções negativas ativadas em situações desagradáveis (down regulation). A análise fatorial confirmatória indicou que o modelo de dois fatores se ajusta melhor aos dados da amostra brasileira, oferecendo um indicador robusto de validação da estrutura bifatorial da medida. Além disso, os resultados da análise da versão reduzida do instrumento também apresentaram bons índices de ajustamento e estes se mostraram melhores do que os de uma solução em que todos os cenários saturavam num único fator geral.

Os resultados da medida permitem assegurar que o ERP pode indicar a frequência de processos regulatórios individuais, diferenciando pessoas que tendem a potencializar as emoções positivas (up regulation) mais que reduzir a intensidade de emoções negativas (down regulation), daquelas que tendem a focar seus processos regulatórios na redução dos malefícios das emoções negativas, quando ativadas, dando menos importância à potencialização de emoções positivas desencadeadas por situações agradáveis.

A versão reduzida representa um contributo importante para a pesquisa sobre a regulação emocional porque facilita a realização de estudos que venham a fazer uso de outros instrumentos para analisar o papel desempenhado pela regulação emocional noutras variáveis. A versão com- 
Gondim, S. M. G., Pereira, C. R., Hirschle, A. L. T., Palma, E. M. S., Alberton, G. D., Paranhos, J., Santana, V. \& Ribeiro, W. R. B. (2015), Evidências de Validação de uma Medida de Características Pessoais de Regulação das Emoções.

pleta com 15 cenários é demasiado longa, o que aumenta a dificuldade de sua aplicação conjunta com medidas adicionais, fundamentais em estudos que visam ampliar a compreensão da interação da regulação emocional com outros construtos.

A despeito de se reconhecer que a medida do ERP representa uma contribuição importante para a literatura sobre os processos regulatórios visto que coloca em saliência o contexto em que uma emoção específica é ativada, uma de suas limitações é a de fazer apenas uma avaliação retrospectiva de como a pessoa hipoteticamente agiria em cada contexto. Isto ocorre porque o ERP mede a regulação emocional por meio da apresentação de situações hipotéticas ativadoras de emoções positivas ou negativas, avaliando somente a percepção individual. A mobilização de repertórios regulatórios que pode ter lugar em contextos naturais, exigindo manejo emocional espontâneo, de fato ainda é pouco estudada. Induzir artificialmente o contexto para a regulação ocorrer e avaliar retrospectivamente o uso de estratégias ignorando a situação, o tipo de emoção e o contexto, limita o conhecimento sobre este importante processo psicológico.

Outra limitação do ERP reside no reduzido número de aspectos regulatórios avaliados, centrando-se em apenas dois mecanismos: down regulation, para emoções negativas e up regulation, para emoções positivas. São desconsideradas as diferenças individuais no uso de outras estratégias, ou mesmo a possibilidade de combinação entre diferentes estratégias. Em outras palavras, duas pessoas podem ter o mesmo perfil regulatório, ao fazer mais uso da down regulation do que da up regulation, mas difiram no uso de estratégias funcionais (modificação da situação, reorientação da situação, reavaliação positiva da situação negativa, expressão de emoções negativas) e disfuncionais (desamparo aprendido, ruminação, abuso de substâncias e reação impulsiva-acting out). Na maneira como o escore é computado pelos autores proponentes da medida, essas diferenças são desconsideradas.

Certamente ainda há muito que trilhar na construção de boas medidas psicológicas para estimular o campo de estudos da regulação emocional. Embora o ERP não atenda a todas as demandas que exigem o campo de estudos da regulação emocional, oferece bons indicadores para serem usados como uma medida de avaliação da frequência de tipos de regulação em situações cotidianas, em que estejam envolvidos o aumento dos efeitos benéficos das emoções positivas quando ativadas e a diminuição dos malefícios das emoções negativas quando ativadas para fins de preservação de bem-estar pessoal.

\section{Referências}

Aldao, A. (2013). The future of emotion regulation research: Capturing context. Perspectives on Psychological Science, 8(2), 155-172. doi:10.1177/1745691612459518

Borsa, J. L., Damásio, B. F., \& Bandeira, D. R. (2012). Adaptação e validação de instrumentos psicológicos entre culturas: Algumas considerações. Paidéia (Ribeirão Preto), 22(53), 423-432. doi:10.1590/1982-43272253201314
Byrne, B. M. (2010). Structural equation modeling with AMOS: Basic concepts, applications, and programming. Mahwah, NJ: Lawrence Erlbaum.

Cassepp-Borges, V., Balbinotti, M. A. A., \& Teodoro, M. L. M. (2010). Tradução e validação de conteúdo: Uma proposta para a adaptação de instrumentos. In L. Pasquali (Ed.), Instrumentação psicológica: Fundamentos e práticas (pp. 506-520). Porto Alegre, RS: Artmed.

Diefendorff, J. M., Croyle, M. H., \& Gosserand, R. H. (2005). The dimensionality and antecedents of emotional labor strategies. Journal of Vocational Behavior, 66, 339-357. doi:10.1016/j. jvb.2004.02.001

Fabes, R. A., Eisenberg, N., Jones, S., Smith, M., Guthrie, I., Poulin, R., ...Friedman, J. (1999). Regulation, emotionality, and pre-schoolers' socially competent peer interactions. Child Development, 70, 432-442.

Grandey, A. A. (2000). Emotion regulation in the workplace: A new way to conceptualize emotional labor. Journal of Occupational Health Psychology, 5, 95-110. doi:10.1037/10768998.5.95

Gross, J. J. (1998). The emerging field of emotion regulation: An integrative review. Review of General Psychology, 2, 271-299. doi: 10.1037/1089-2680

Gross, J. J., \& Thompson, R. (2007). Emotion regulation: Conceptual foundations. In J. J. Gross (Ed.), Handbook of emotion regulation (pp. 3-24). New York: Guilford.

Hochschild, A. R. (1983). The managed heart: Commercialization of human feeling. Los Angeles, CA: University of California Press.

Izard, C. E. (2010). The many meanings/aspects of emotion: Definitions, functions, activation, and regulation. Emotion Review, 2(4), 363-370. doi:10.1177/1754073910374661

Izard, C. E., Woodbum, E. M., Finlon, K. J., Krauthamer-Ewing, E. S., Grossman, S. R., \& Seidenfeld, A. (2011). Emotion knowledge, emotion utilization, and emotion regulation. Emotion Review, 3(1), 44-52. doi:10.1177/1754073910380972

Mennin, D. S. (2006). Emotion regulation therapy: An integrative approach to treatment-resistant anxiety disorders. Journal of Contemporary Psychotherapy, 36, 95-105.

Nelis, D., Quoidbach, J., Hansenne, M., \& Mikolajczak, M. (2011). Measuring individual differences in emotion regulation: The Emotion Regulation Profile-Revised (ERP-R). Psychologica Belgica, 51, 49-91. doi:10.5334/pb-51-1-49

Níven, K., Totterdell, P., Stride, C. B., \& Holman, D. D. (2011). Emotion Regulation of Others and Self(EROS): The development and validation of a new individual difference measure. Current Psychology, 30, 53-73. doi:10.1007/s12144-0119099-9

Pasquali, L. (2010). Instrumentação psicológica. Fundamentos e práticas. Porto Alegre, RS: Artmed.

Zhao, B. (2011). Learning from errors: The role of context, emotion, and personality. Journal of Organizational Behavior, 32 , 435-463. doi:10.1002/job.696 